\title{
Factors affecting the acceptability and consumption of Corn Soya Blend Plus as a prenatal dietary supplement among pregnant women in rural Cambodia
}

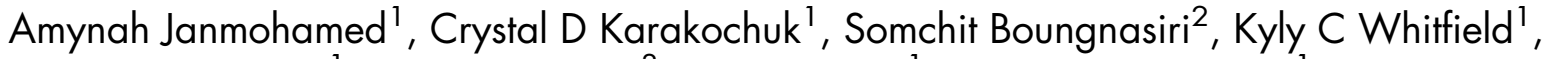 \\ Gwen E Chapman ${ }^{1}$, Patricia Janssen ${ }^{3}$, Judy McLean ${ }^{1}$ and Timothy J Green ${ }^{1, *}$ \\ ${ }^{1}$ Food, Nutrition and Health, The University of British Columbia, 215-2205 East Mall, Vancouver, BC, Canada, \\ V6T 1Z4: ${ }^{2}$ International Relief and Development, Cambodia Country Office, Phnom Penh, Cambodia: ${ }^{3}$ School of \\ Population and Public Health, The University of British Columbia, Vancouver, BC, Canada
}

Submitted 3 April 2015: Final revision received 21 July 2015: Accepted 18 September 2015: First published online 16 0ctober 2015

\begin{abstract}
Objective: Undernutrition is prevalent among pregnant women in Cambodia. The provision of fortified dietary supplements is one strategy to help pregnant women meet their nutritional needs. Corn Soya Blend Plus (CSBP) is a widely used prenatal dietary supplement in areas with high rates of undernutrition and food insecurity. However, little is known about its acceptability during pregnancy. The present study aimed to identify factors that affected the acceptability and consumption of CSBP supplements among pregnant women.

Design: Women completed a structured interview designed to provide information on facilitators of and barriers to utilization. In addition, six focus groups were conducted with a subset of women $(n 70)$ to further explore attitudes, perceptions and experiences related to CSBP use.

Setting: Two districts in Kampong Chhnang Province, Cambodia.

Subjects: Pregnant women ( $n$ 288) participating in a cluster-randomized trial of CSBP.

Results: The acceptability of CSBP was influenced by sensory attributes, family support, peer influences, and attitudes related to diet, nutritional status and weight gain in pregnancy. Attaining adequate nutrition was considered less important than other concerns during pregnancy, particularly anxiety related to the costs of delivery and postpartum care. Acceptance was lower among new mothers due to fears of weight gain. Health benefits were common reasons for continued use and minor side-effects, such as nausea, were not major barriers to consumption.

Conclusions: CSBP was generally well accepted in this population. However, organoleptic factors and perceptions regarding nutrition and weight gain in pregnancy, particularly for first-time mothers, were barriers to increasing acceptance among Cambodian women.
\end{abstract}

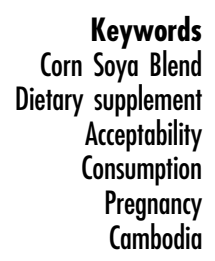

Attaining adequate energy and nutrients during pregnancy is critical for optimal maternal and newborn health outcomes $^{(1)}$. Currently, Fe and folic acid supplementation is the only nutritional intervention recommended for all pregnant women by the $\mathrm{WHO}^{(2)}$. At present, evidence is lacking to justify interventions to reduce protein-energy and other micronutrient deficiencies, with the exception of Ca supplementation for pre-eclampsia prevention in areas where Ca intake is low ${ }^{(3)}$.

Undernutrition is one of Cambodia's most pressing challenges. Recent data indicate a serious problem of food insecurity in the country ${ }^{(4)}$ and the prevalence of chronic child undernutrition is among the highest in Southeast Asia $^{(5)}$. Further, a large proportion of women of childbearing age are undernourished and over half of women are anaemic during pregnancy ${ }^{(6)}$. While some progress has been made towards reducing early child undernutrition in recent years, mainly as a result of national breast-feeding and complementary feeding programmes, maternal undernutrition remains a problem ${ }^{(6)}$. Recommendations for pregnant women in Cambodia include consumption of an extra daily meal and gestational weight gain of at least $7 \mathrm{~kg}^{(7)}$. However, the staple food in Cambodia, white rice, has low energy density, poor 
protein quality and provides inadequate micronutrients, which prevents many women in Cambodia from achieving these goals ${ }^{(8)}$. The burden of undernutrition is especially high among women and children in Kampong Chhnang Province where the prevalence of anaemia among women of childbearing age is $57 \%$, which is $13 \%$ higher than the national average and the second highest in the country ${ }^{(6)}$. Stunting (low height-for-age) among children $<5$ years of age in the province is $40 \%$ and the prevalence of anaemia in this age group is $64 \%$, the third highest in the country ${ }^{(6)}$.

Reducing maternal and child undernutrition during the first $1000 \mathrm{~d}$ between conception and 24 months is a key priority of donor and development agencies ${ }^{(9)}$. One approach is to provide targeted food supplements, such as fortified blended foods, to pregnant women and other nutritionally vulnerable populations through food assistance programmes ${ }^{(10)}$. Such foods aim to supplement the local diet with essential macro- and micronutrients. The energy-dense, micronutrient-fortified maize and soyabean flour, Corn Soya Blend Plus (CSBP), is commonly used in supplementary feeding programmes for pregnant women $^{(11)}$, although little is known about its acceptance in this population. Currently, there are no global recommendations for the use of CSBP as a prenatal nutritional supplement and product distribution is conditioned on donor programming. For dietary supplements such as CSBP to improve the nutritional status of beneficiaries, they must first be acceptable in the populations they are used. This entails exploring factors that facilitate or challenge their uptake. This gap in knowledge is significant and has important resource and ethical implications for both suppliers and recipients of these supplements. To our knowledge, there are no published studies on the acceptability of CSBP among pregnant women.

The present study was embedded in a CSBP efficacy trial conducted between August 2011 and March 2013, in which women received the supplement monthly starting in the first trimester through the final month of pregnancy (unpublished data; ClinicalTrials.gov NCT01413776). In the trial among 547 pregnant women in Kampong Chhnang Province, CSBP was effective for reducing maternal anaemia in late gestation and for reducing the rate of preterm birth, but did not confer significant benefits on maternal weight gain or birth size. We investigated factors that affected the acceptability and consumption of CSBP among women who participated in the trial. The findings of the acceptability study provide valuable insight that can be used to guide future programming and policy on the provision and distribution of dietary supplements in Cambodia. Further, the findings highlight potential factors that may also be inhibiting optimal acceptance, consumption and, thereby, the impact of CSBP in other geographical regions and among other population groups.

\section{Materials and methods}

\section{Participant selection}

The efficacy study was a two-arm cluster trial in which seventy-five villages (clusters) were randomly assigned to receive the treatment (daily ration of $200 \mathrm{~g} \mathrm{CSBP}$ and $10 \mathrm{ml}$ vegetable oil) or to a control group. During each month of pregnancy, women were provided approximately $7 \mathrm{~kg}$ of CSBP in durable plastic bags provided by the World Food Programme, the donor of the supplement. CSBP was distributed to women by study personnel with the assistance of trained female village health workers. The distribution process was closely monitored to prevent product leakage by requiring the recipient's signature on a delivery form and through spot checks by the study team to verify women received their supplements in a timely manner. At the enrolment visit at the household, women were introduced to CSBP using a flipchart that illustrated the product's purpose and instructions for storage, cooking and consumption. Women were advised to cook the CSBP and oil together as a porridge. Family members (husbands, parents, children) present in the household were encouraged to listen to the education messages and cooking instructions so they could help prepare it if necessary.

The study area included primarily rice-farming communities and had an average village population size of 660. In Cambodia, rice constitutes the main part of the meal and a commonly used expression is 'if a person has not eaten rice, a person has not eaten'. Small amounts of vegetables and fish or meat (if available) are typically eaten with rice, but are not substantive components of the meal. The importance of rice in the country's food culture is also reflected in the Khmer language in which 'eating' is literally translated as 'eating rice ${ }^{\text {,12) }}$. In the efficacy study, $40 \%$ of women had a low BMI $\left(<18.5 \mathrm{~kg} / \mathrm{m}^{2}\right)$ and approximately $30 \%$ were anaemic $(\mathrm{Hb}<11 \mathrm{~g} / \mathrm{dl})$ in the first trimester of pregnancy. Women were eligible for the trial if they were at least 18 years of age, had an antenatal care visit in the first trimester of pregnancy and intended to remain in their home village for the duration of pregnancy. Study outcomes evaluated were maternal weight gain, maternal anaemia status at four time periods during pregnancy, newborn anthropometry and duration of gestation. The design, methods, participant characteristics and results of the trial are under consideration elsewhere.

For the acceptability study, which was nested within the larger trial, structured interviews and focus groups were conducted. The structured interviews were conducted with all women in the treatment group who completed the trial and were administered individually at the household following delivery. Six focus groups were conducted with a subset of women in the CSBP (treatment) group after delivery ( 1-6 months after delivery). As the number of trial participants from each village was small, all women who completed the trial were eligible to participate in their respective village's focus group. Focus group villages were 
purposively selected based on geographic representation and diversity of experience in an attempt to achieve maximum sample variation. As the acceptability study was embedded in the larger trial, the informed consent procedure for the trial included possible participation in the qualitative study. The trial and nested qualitative study were approved by the Cambodian National Ethics Committee for Health Research and the University of British Columbia Clinical Research Ethics Board (H11-00801). No participants were excluded on the basis of ethnicity, religious affiliation or socio-economic status, and all aspects of data collection, management, storage and dissemination safeguarded the confidentiality of study participants.

\section{Data collection, management and analysis}

Methods for data collection included structured interviews and focus group discussions (FGD). We used an explanatory sequential design ${ }^{(13)}$ in that the interviews and FGD were administered and analysed sequentially but, for the purposes of the current paper, the findings are integrated to more broadly address the research question. Through these two methods, we were able to fully explore the CSBP user experience. The questionnaire for the structured interview included primarily closed-ended questions and was designed to collect information on behaviours pertaining to supplement usage (cooking practices, consumption preferences, household sharing), the amount women consumed, and benefits and adverse effects women associated with consuming the supplement. We used open-ended questions to elicit suggestions for product improvement. The interview guide was pre-tested in the local community and revisions consisted mainly of terminology changes.

The FGD were designed to expand on aspects of the interview (user preferences) and investigate in greater depth the factors that positively and negatively affected uptake of the CSBP intervention. Through the FGD, we sought to gain a deeper understanding of and explanation for the supplement-related behaviours observed in our study population. We utilized focus groups instead of indepth interviews given the advantage of this methodology to identify prevailing cultural/community norms and shared beliefs ${ }^{(14)}$ and because we wanted to capture the rich data inherent in inter-participant interactions and behaviours fostered in a group discussion setting. As Kitzinger notes, 'Focus groups reach the parts that other methods cannot reach, revealing dimensions of understanding that often remain untapped by more conventional data collection techniques, ${ }^{,(15)}$. Furthermore, based on local input and circumstantial knowledge, we felt group discussions would engage women more fully in this cultural context. Table 1 presents the key topic areas explored in the interviews and focus groups.

Both the structured interviews and focus groups were conducted by International Relief \& Development (IRD)
Table 1 Key topics in the structured interviews and focus group discussions with women ( $n$ 288), Kampong Chhnang Province, Cambodia

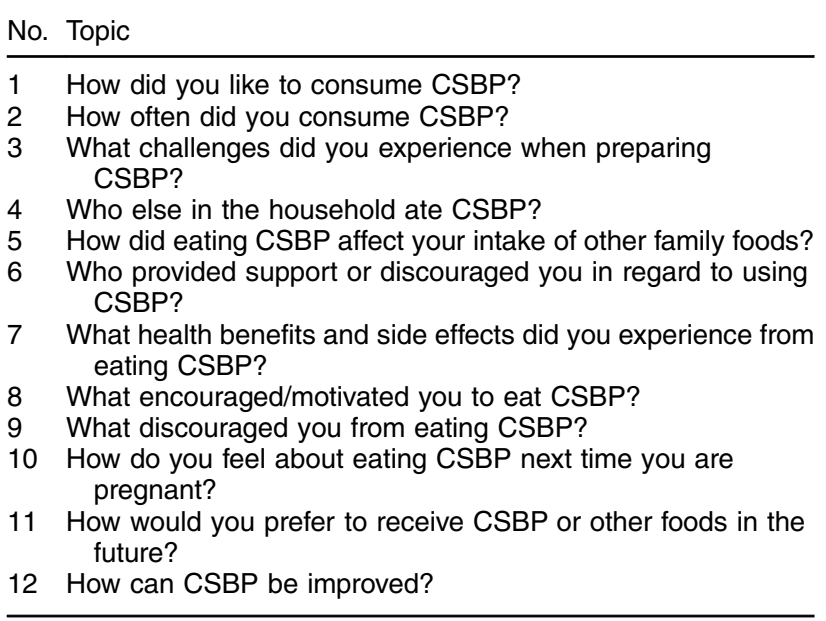

CSBP, Corn Soya Blend Plus.

research officers who were trained by senior researchers and technical experts from a large multi-year child survival project. The interviews took place at women's homes within one month of delivery and took an average of $45 \mathrm{~min}$ to complete. Participant answers were recorded in writing by the interviewer and forms were checked by the research supervisor for completeness and obvious recording errors, which were corrected. Automated quality control checks were built into the data entry process. Data obtained from the structured interviews were analysed quantitatively for the entire sample using simple descriptive methods and are presented as frequencies and percentages in the current paper.

FGD were conducted in each selected village after all women in the respective village delivered. They were held at the home of a village health volunteer, the village pagoda or other convenient meeting place and lasted 90 to 120 min. The FGD guide contained key questions to direct the discussion, but allowed flexibility to explore topics as they emerged. Probing techniques were used as necessary to explore ideas and viewpoints in further detail. Each audio-taped focus group was facilitated by one moderator and two note-takers captured nuanced aspects of the discussions, including participant disposition, tone and emotion. The moderator and note-takers prepared a detailed report of each discussion in the local language from the audio-recording and expanded field notes, and then produced a synthesized summary in English. The summaries were assessed for completeness, accuracy and quality of translation by bilingual IRD project staff who were external to the study team. The first author (A.J.) and the study facilitators performed a thematic content analysis on the synthesized summaries. This entailed organizing text around relevant themes and sub-themes that emerged during the discussions using an inductive coding scheme. 
The method allowed for iterative coding as we gained more in-depth understanding of the data. Coded segments were manually grouped and inserted into textual matrices in MicroSoft ${ }^{\circledR}$ Word for interpretation. We used the constant comparison method ${ }^{(16)}$ to examine findings of each focus group in relation to preceding discussions. Illustrative quotes of participants, expressed during interviews and FGD, are included herein to reinforce key points and concepts raised. Perspectives of 'deviant' cases, that is, opinions expressed by one or very few individuals, were described for completeness.

Our analytical approach entailed organizing data based on a framework developed by Young et al. in their acceptability study of three nutritional supplements among pregnant and lactating women in Mexico ${ }^{(17)}$. This grounded-theory approach involved examining three aspects of acceptability: organoleptic properties, ease of use, and positive and negative health effects. We chose to adapt this conceptual scheme as the study closely paralleled our research in terms of the aspects of acceptability investigated and it was also conducted in the context of a cluster-randomized efficacy trial that evaluated the impact of the three supplements on maternal nutrition outcomes. In addition to Young et al.'s three thematic categories, our framework included an exploration of women's attitudes and perceptions towards CSBP as we believed these to be important determinants underlying its use and acceptance in our study context. Here, data from structured interviews and focus groups are combined and presented together according to the components of the conceptual framework: organoleptic qualities, feasibility of use, health effects, and attitudes and perceptions. The data source (interview or FGD) is specified, where relevant, to provide further clarity. All data entry, management and analyses for the CSBP acceptability study were conducted locally at the IRD office premises in Kampong Chhnang, Cambodia.

\section{Results}

A total of 288 women from thirty-seven villages in Kampong Chhnang Province participated in the structured interview, in addition to seventy women from six villages who also participated in the FGD component. All women in the treatment intervention group who completed the efficacy trial participated in the interview. The average age of participants was 26 years and, although most had some formal schooling, $40 \%$ did not complete primary school (grade 6). Most households (>70\%) were classified as impoverished, according to the World Bank definition of living on less than $\$ \mathrm{US} 2 / \mathrm{d}^{(18)}$. The majority of women $(\sim 70 \%)$ were rice farmers and relied on one annual harvest. Similar to other regions of Cambodia, the typical diet in the study area was of low nutritional quality, containing few bioavailable sources of key micronutrients ${ }^{(8)}$.
On average, women began receiving CSBP during week 8 of gestation and the average duration of supplementation was 30 weeks. Monthly mean and median consumption was 18 rations. Women were asked to keep daily CSBP consumption records that were collected by field researchers on a weekly basis and consumption data were also obtained during the structured interviews. The amount consumed was based on the quantity of flour women cooked for themselves each day. Intake of CSBP was not supervised at the household.

\section{Organoleptic qualities}

Women were very sensitive to the organoleptic qualities of CSBP, particularly the sensory characteristics of smell and taste. Almost all women (>90\%) disliked the smell produced during the boiling process, which they referred to as an undesirable 'corn smell', and the majority said the taste was bland and flavourless. Women's aversion to these attributes of CSBP was particularly acute during periods of morning sickness, which more than half of women experienced beyond the first trimester:

'I was having morning sickness and the smell when cooking [CSBP] porridge made me feel sick in my stomach and I had to vomit. It would be better if it did not smell when cooking.'

In the FGD, numerous women mentioned adding sugar to make the supplement more palatable, although some said having to purchase extra sugar meant they could consume limited quantities of CSBP:

'I could not eat it [CSBP] the way it was given to us because the taste was not good. I had to go to the market to buy sugar so I could eat it because they told me it was good for my baby. But I could not keep buying more sugar because my family is poor so I did not eat a lot.'

Aside from adding sweetness to improve the taste of the porridge, women cooked the flour in other ways such as frying, making a beverage and preparing a thick mush, which was translated to mean 'cake'. Only a few women stated they liked the CSBP as it was provided to them, as illustrated by one woman's comment:

'It was nice to eat when I roasted it on the fire. In the village we are poor. IRD gave us this food and we are happy. We never got food before.'

\section{Feasibility of use}

Information obtained from interviews and focus groups revealed no major logistical or structural barriers to incorporating CSBP into women's daily routines. Preparatory requirements in terms of time, additional cooking fuel and availability of water for boiling did not affect women's willingness or ability to prepare the food 
supplement. Most women felt it was easy to cook and reported an average cooking time of 12 to $13 \mathrm{~min}$, which was considered minimal. One-quarter of participants said family members, particularly mothers and husbands, assisted with cooking during times of illness, fatigue and on days when women were too busy. This created an enabling environment for uninterrupted consumption:

'When I was close to delivery and did not have energy in my body, my mother told me I had to eat the vitamin food [CSBP] to be ready and she was cooking it for me.'

In the FGD, it became apparent that many women found it challenging to store the flour in the plastic bags in the household because they were not airtight once opened and were, therefore, easily penetrated by insects, rodents and other pests. Instead, they said it would be better to receive CSBP in a reusable plastic or tin container that could be refilled each month. A few women said smaller quantities provided on a more frequent basis (weekly or fortnightly) would be preferable for easier home storage and management. Women were generally satisfied with the quantity of CSBP they received. However, based on consumption records maintained by women and field observations by research officers, intake was less than maximum as women were not consuming the entire daily ration $(200 \mathrm{~g})$. Reasons women gave for this included the large ration size and preference for consuming it as a snack between meals, rather than as the intended extra porridge meal, which is presumably rooted in traditional eating practices. Other reasons given for the popularity of CSBP snacks were women's preferred cooking methods alluded to earlier (frying, beverage), which were more conducive to being eaten in small amounts rather than as full meals. Finally, CSBP was not incorporated into the daily meal as only $2 \%(n 5)$ of interview respondents said they consumed it with their normal 'family' foods. This was due to preference and not to avoid sharing it at mealtimes.

As the food supplement was specifically targeted to pregnant women in the household, and most Cambodian households are multi-generational, we explored women's decision making with regard to dietary choices and eating habits during pregnancy. Encouragingly, all women interviewed stated they were responsible for their food choices and controlled their eating behaviours, including consumption of CSBP, when they were pregnant. Moreover, the FGD further revealed that the presence of an additional food source in the household did not exert any untoward pressures on women to cook it for their husbands and/or parental elders. Alternative uses such as feeding it to their children were voluntary choices made by few women. Family members were generally supportive and encouraged women to cook and eat the CSBP, which facilitated utilization. In the interviews, all women said their husband approved of them consuming the supplement.
We encountered mixed opinions regarding preferred forms of food supplements. Despite the undesirable flavour, more women liked having the uncooked flour because it could be customized to taste and cooking style. Such women said if they received a ready-to-eat food and did not like it, nothing could be done and it would be wasted:

'If they give us something else that tastes bad and we cannot change the taste, we cannot do much. With this [CSBP] even though we do not like the taste much we can make it better.'

Fewer women said a ready-to-eat food would be more convenient because it would not require cooking, could be eaten anywhere, and that a packaged product would be easier to store in the home:

'It would be better to have something we can eat any time and take to the [rice] field.'

Although $99 \%$ of women interviewed expressed willingness to receive CSBP during a future pregnancy if offered at no cost and delivered to them directly as occurred during the trial, fewer ( $80 \%$ ) said they would be willing to collect it from the nearest health centre, which we used as a proxy point of distribution to gauge product demand. Willingness to pay for CSBP was not determined, given that the product is currently only provided at no cost as part of donor-led global food assistance programmes.

\section{Health effects}

In the structured interviews, 99\% of women reported at least one perceived benefit from consuming CSBP. Benefits were primarily associated with the mother's health and well-being during pregnancy and the size, strength and general health of the newborn (Table 2):

'After eating the food [CSBP], I had more energy. With my last child, I did not have much energy and felt dizzy and had to sleep a lot.'

One woman said the CSBP enabled her to produce more milk during lactation, compared with her previous breastfeeding experience. Specific to the newborn, positive effects mentioned were a healthy, normal-weight and pretty baby and the infant being ill less frequently. In the FGD, a few women cited indirect health benefits such as being able to take their other children to the health centre and buy medicines because they were able to save some money due to having the additional CSBP food source. Other benefits mentioned by women during the group discussions were rapid infant growth and that their baby was clever, which was determined to mean active in the local language.

Negative effects of CSBP were reported by about $10 \%$ of interview respondents and consisted of adverse events experienced by the women. Diarrhoea and nausea were the most common complaints. Three women reported stomach pain, one woman experienced dizziness, one reported headache and one said CSBP induced a fever. The majority 
Table 2 Reported benefits and adverse effects of CSBP in pregnancy among women ( $n$ 288), Kampong Chhnang Province, Cambodia*

\begin{tabular}{lrr}
\hline & $n$ & $\%$ \\
\hline Reported benefit to mother (N 282) & & \\
Felt healthier & 204 & 72 \\
Had more energy & 189 & 67 \\
Increased appetite/gained weight & 9 & 3 \\
Had easy delivery & 4 & 1 \\
Produced more breast milk & 1 & $<1$ \\
Saved money & 1 & $<1$ \\
Reported benefit to baby (N 282) & 210 & 75 \\
Born with healthy/normal weight & 42 & 15 \\
Was not ill frequently/grew well & 22 & 8 \\
Born with pretty skin & 15 & 50 \\
Reported adverse effect to mother (N 30) & 7 & 23 \\
Diarrhoea & 3 & 10 \\
Nausea & 1 & 3 \\
Abdominal pain & 1 & 3 \\
Dizziness & 1 & 3 \\
Fever & & \\
Headache & & \\
\hline
\end{tabular}

CSBP, Corn Soya Blend Plus.

${ }^{*}$ Data from structured interviews; $N$ denotes total number of women who reported a benefit ( $N$ 282) and an adverse effect $(N 30)$.

of women said side-effects lasted a short time and were not bothersome. Three women said they experienced sideeffects often, or on a daily basis, and only one woman reported not being able to eat any CSBP due to continuing nausea and stomach upset. In general, side-effects did not appear to be a barrier to consumption of the supplement:

'The first week I ate it [CSBP] I had a pain in my stomach. I asked the VHV [village health volunteer] and she told me it would go away and I should try eating it again because it is good for my baby. I did what she told me and the pain got less.'

Table 2 presents the health benefits and adverse effects women associated with consuming the supplement.

\section{Attitudes and perceptions}

Exploration of women's attitudes and perceptions in the focus groups revealed two dominant themes related to acceptance of CSBP: women's perspectives on being undernourished during pregnancy and views towards gestational weight gain. In the present study context, nutritional adequacy held less importance than other concerns women had during their pregnancy. Lacking money for the cost of delivery and necessary supplies for the newborn was a major source of anxiety. Women said they often compromised on food purchases to save money for childbirth expenses. Some women expressed regret about not being able to have a better diet:

'I wanted to eat stronger food like beef, but I had to keep the money for delivery at the health centre and for the blanket, bottle and clothes for my baby.'

Women's opinions about being undernourished in pregnancy were also reflected in the relative homogeneity between the pre-pregnancy and prenatal diet. In general, having good health was associated with eating 'enough'. Therefore, eating well in pregnancy typically meant increasing intake of routinely consumed foods, namely rice, as opposed to seeking out higher-quality proteins such as meat and eggs. This was also due, in large part, to economic constraints that limited access to such costlier foods. The emphasis on food quantity was not only circumstantial, but was also influenced by messages typically provided during routine antenatal care sessions. At these visits, women were encouraged to eat more, though were provided little guidance on intake of specific foods, aside from leafy green vegetables which were strongly emphasized as dietary sources of Fe. CSBP acceptance was affected by women's perceived household food availability. Women who had reported having enough food, irrespective of quality, consumed less CSBP, while those living in households with less food available showed greater acceptance. In general, dietary replacement with CSBP was not a common practice.

The second dominant theme that emerged surrounding women's attitudes towards the food supplement related to the topic of weight gain in pregnancy. Women who were pregnant for the first time were less receptive to the CSBP due to fears of weight gain, a large fetus and resulting delivery complications. Some underlying stigma surrounding being 'cut', that is, having a caesarean section, was inferred from some of the FGD and could have contributed to these fears. Although women said they primarily consulted midwives for trusted advice during pregnancy, it became apparent that counselling on the risks of inadequate gestational weight gain was not standard practice. New mothers feared the unfamiliar childbirth experience and labour pain due to their tighter pelvic muscles. These fears influenced their willingness to consume a product that was partly promoted to increase maternal weight gain and baby weight. In contrast, women who had other children more readily accepted the CSBP because they believed it would help them build up vitamins and 'power' (energy) for delivery, which they wanted based on their past childbirth experience. Also, several women said they did not want an underweight and weak baby with frequent illness as this would incur unmanageable costs for health centre visits and medicines:

'My last child was born very small. The midwife said the baby had no power to drink mother's milk and grow well. I took my baby to hospital and they took much money from me. This time I ate more and the new food [CSBP] to make my baby grow enough inside.'

A few women were concerned about having a child with a deformity and some believed the vitamins in the supplement would prevent a malformation:

'I ate the CSBP because I was afraid my baby would not have a full body.' 
These divergent opinions regarding weight gain in pregnancy were reflected in how CSBP was incorporated into women's eating practices. For women who were eager to gain weight, eating CSBP fit with other weightpromoting practices such as increasing portion sizes, having more frequent meals and snacks, and consuming high-energy foods. For women who wanted to control their weight gain, eating less CSBP was accompanied by weight-limiting behaviours such as reducing food intake and frequency and avoiding foods such as sweets and other foods associated with weight gain.

We explored the level of intra- and inter-household sharing among CSBP recipients. Although about $80 \%$ of women reported sharing the supplement within the household in the interview, two-thirds said they only shared the equivalent of one or two rations per month. Seven (3\%) interview respondents reported sharing it on a daily basis. Distribution outside the household was less common ( $20 \%)$ and quantities allotted to neighbours and/or relatives were similarly small. Selling CSBP was not reported or observed in this setting. Individual interpersonal messaging and the package label targeted only to pregnant women likely discouraged household sharing. Finally, the knowledge and trust instilled through frequent interactions with the IRD study team, positive experiences of co-participants recruited earlier in the study (e.g. more energy, smooth delivery, healthy baby, pretty baby), as well as safety assurances provided through peer experiences cultivated positive attitudes towards CSBP and motivated later recruits:

'At first I was not sure about it [CSBP], but then I saw my neighbour eat it and she had a healthy and clever baby so I thought to eat it also.'

In general, women enrolled later in the study were more motivated as they had developed an awareness and interest in the CSBP and perceived notions of safety and benefit. Also, these women had more recipes to draw from their neighbours. Lastly, CSBP was not associated with negative consequences other than women's perceived problems associated with excess weight gain. For example, it was not likened to a prevailing food taboo or traditional food avoidance in pregnancy. Therefore, such culturally imposed potential barriers to consumption were unlikely.

\section{Discussion}

In the present study we explored women's experiences with CSBP supplements during pregnancy in a rural Cambodian setting. Acceptance of the dietary supplement was explored through women's beliefs about palatability, ease of preparation, health effects, and attitudes and perceptions that motivated or inhibited use. Using the two data collection methods, we were able to obtain representative data on CSBP cooking and eating practices for the entire sample in the interviews and to contextualize consumption in the broader behavioural context with respect to other pregnancy behaviours identified through the group discussions.

Although sensitivity to the product's taste and smell affected uptake, logistical considerations such as additional time and cooking fuel did not discourage preparation. This differs from the literature on infant and young child complementary feeding programmes which suggests a mother's lack of time is often a barrier to preparing special foods for children and nutrition supplements requiring active preparation are typically less well accepted than ready-to-use foods ${ }^{(19)}$. CSBP adherence was situated within the context of maternal nutrition being less important than other concerns requiring protective action such as the cost of health centre visits, delivery and postpartum care and amidst fears of gestational weight gain among new mothers. Parallels can be drawn between our findings and the literature on interventions to reduce malaria in pregnancy, which suggests vulnerability to illness should be viewed in relation to other needs and concerns women have during pregnancy and that perceived severity of illness can be a strong catalyst for uptake of preventive interventions ${ }^{(20)}$. This can be seen in the similarity of the maternal diet prior to and during pregnancy in our population and has also been observed in Burkina Faso ${ }^{(21)}$, India ${ }^{(22)}$ and $\operatorname{LaOs}^{(23)}$.

Positive attitudes, conceived experientially by women who had previously undergone childbirth and cared for a weak and frequently ill newborn, were reflected in weightpromoting practices including willingness to consume the dietary supplement. In contrast, fears of a large baby and difficult labour and delivery reduced acceptance of CSBP among many first-time mothers. Similar concerns and purposive reductions in food intake during pregnancy have been identified in other settings, including Bangladesh and Indonesia ${ }^{(24,25)}$. A study among Somali immigrants in Sweden identified negative attitudes towards 'abnormal' caesarean delivery and decreased food intake to avoid the procedure ${ }^{(26)}$. Our findings suggest women's views towards weight gain in pregnancy can considerably influence acceptance of a food supplement and should be considered when introducing interventions that promote energy, weight gain and increased birth size. Additionally, specific messages should cater to especially vulnerable new mothers who tend to be younger and undernourished. In our study, women's control over their food choices in pregnancy facilitated acceptance of CSBP in the household. This is an important finding and points to the merits of targeting women directly with health and nutrition messages, while creating a supportive family environment to reinforce behaviours. This contrasts to a study conducted by Shannon et al. in Bangladesh which revealed the majority of food reduction in pregnancy was due to intra-household food allocation practices that disproportionately affected women ${ }^{(24)}$. Furthermore, nutrition-focused counselling should be a 
core component of antenatal care services as midwives were considered the most trusted source for advice and care in pregnancy in our study context.

Despite the fact that most women disliked the taste and smell of CSBP, almost all reported a health benefit, either for themselves or their baby, which they attributed to the supplement. This finding was also observed in a study involving a ready-to-use therapeutic food in Bangladesh where health benefits in pregnancy were reported in a context of low supplement acceptability ${ }^{(27)}$. In our study population, CSBP was considered a food and not a medicine, although it was promoted as a source of vitamins and minerals. This likely contributed to lower acceptance among those fearing weight gain because food is typically associated with body size, and to increased acceptance among others as it was considered a healthy food and not a medicinal product with an unknown safety profile. This finding is consistent with Young et al.'s study among rural Mexican women that partly attributed acceptance of a prenatal fortified powder beverage to it being perceived as a food, rather than a medicine, as compared with the other (tablet and micronutrient powder) interventions evaluated in the trial ${ }^{(17)}$. The practice of sharing CSBP was not as widespread as we expected. The fact that a large number ( $45 \%$ ) of participants were first-time mothers may partially explain this, as the women did not have other children in the household. However, the possibility of underreporting the extent of household sharing cannot be overlooked.

There is a dearth of evidence surrounding the use of corn-soya-based products in undernourished pregnant women. However, our findings can be compared with field evaluations of Corn Soya Blend in communities in Guatemala, Malawi and Uganda ${ }^{(28)}$. In these contexts, porridge preparations were preferred and foods such as fruits and vegetables were added to make family meals out of the supplement. Contextual variation likely explains these differences. In Uganda and Malawi, beneficiaries were highly dependent on food assistance for their household needs. Also, frequent selling/trading of the product was reported in Uganda, indicating a high value and demand for the product and perhaps higher levels of overall food insecurity. Lastly, corn is a staple food in these countries, unlike in Cambodia, which supported incorporation of the flour into local dishes (e.g. tortillas in Guatemala) and likely contributed to the higher acceptability in these settings. Studies that have explored utilization of other prenatal food supplements have produced mixed results. A study of PlumpyNut ${ }^{\circledR}$ among malnourished pregnant and lactating women in Bangladesh revealed low acceptability due to an unfavourable taste and smell, side-effects and poor understanding of instructions for use ${ }^{(27)}$. In contrast to these findings, lipidbased nutrient supplementation demonstrated high acceptance among pregnant and lactating women in Ghana $^{(29)}$ and in another study in Bangladesh ${ }^{(30)}$.
A strength of our study is that we captured a wide range of CSBP user experiences within a relatively homogeneous socio-economic context. In addition, we assessed acceptability over the duration of pregnancy. Further, all eligible women agreed to participate in the acceptability study. A limitation of this type of research is social desirability response bias ${ }^{(31)}$. As utilization of CSBP was not observed, it is unknown whether participant responses reflected over-reporting of positive opinions and behaviours and a reluctance to provide truthful, albeit negative, opinions for fear of ridicule, intimidation or jeopardizing future opportunities for food or other types of assistance. Also, data were collected after women had delivered and retrospective accounts of attitudes and experiences during pregnancy could have been magnified or attenuated with the elapse of time. This logistical arrangement was made to avoid introducing bias into the efficacy trial and, therefore, could not have been avoided. Furthermore, our findings were generated in the context of a controlled clinical trial in which supplements were provided at the household and frequent interactions occurred between field researchers and participants, which likely contributed to utilization. Acceptability outside a research context should be investigated.

The present study has revealed individual, household, community and institutional factors related to the uptake of CSBP in pregnancy that we speculate are generalizable to other rural settings in Cambodia. The intervention, which was the participants' first exposure to CSBP, was generally well accepted and tolerated, despite aversions to sensory characteristics and dislike of the porridge. We observed greater adherence to CSBP as women derived more confidence and motivation from others' experiences. Local social networks were important for increasing acceptance as they created a level of community support for using the product. Nutrition promotion and allaying fears of weight gain are important programming considerations and provide opportunities for greater adherence. Challenges facing firsttime mothers that may limit supplement consumption necessitate targeted efforts for this vulnerable group. We have presented findings from one geographical and cultural context. Additional studies would be useful to identify factors affecting use of this prenatal dietary supplement in other settings. Food assistance programmes are often criticized for not effectively responding to the nutritional needs of populations. Knowing how and why supplements are used and determining the impediments that challenge their acceptance in different contexts is important for adapting global foodbased programmes to local conditions.

\section{Key messages}

1. Women's perceptions of sensory characteristics and attitudes towards nutrition and weight gain in pregnancy should be addressed to increase acceptance of dietary supplements. 
2. Although CSBP is not a staple food in the local diet of Cambodians, it was generally accepted by pregnant women due to family support, peer influences and the desire for healthy pregnancy and infant outcomes.

3. Understanding how and why supplements are used and the barriers that limit their acceptance in different contexts is important for adapting global food-based programmes to local conditions.

\section{Acknowledgements}

Acknowledgements: The authors gratefully acknowledge the Cambodian Ministry of Health, Kampong Chhnang Provincial Health Department, Boribo Operational District, midwives at Krang Leav, Phsar, Pongro and Svay Chrum health centres, and the local commune and village authorities for their support and collaboration. The authors also acknowledge Meng Kro, Lach Makara, Suorn Sinet and Sary Narong for conducting all field activities, data collection and data entry; members of International Relief \& Development's (IRD) ENRICH project team for their technical, logistical and administrative support; and thank the women who participated in the study. Financial support: This research was funded by the US Agency for International Development's (USAID) Child Survival and Health Grants Program. The World Food Programme donated the CSBP for the study; however, had no role in the study design, data collection, analysis or writing of the manuscript. Conflict of interest: None. Authorship: A.J., J.M. and T.J.G. designed the research; A.J. drafted the research protocol, coordinated and led the research implementation, conducted the data analysis and drafted the research manuscript; S.B., J.M. and T.J.G. provided technical oversight and input into all aspects of the study; C.D.K., K.C.W., G.E.C. and P.J. contributed to the preparation of the manuscript; A.J., J.M. and T.J.G. had primary responsibility for the final content. Ethics of human subject participation: Ethical approval was obtained from the National Ethics Committee for Health Research in Cambodia and the Clinical Research Ethics Board of the University of British Columbia. The trial was registered on ClinicalTrials.gov (NCT01413776).

\section{References}

1. UNICEF (2009) Tracking Progress on Child and Maternal Nutrition. New York: UNICEF.

2. World Health Organization (2012) Guideline: Daily Iron and Folic Acid Supplementation in Pregnant Women. Geneva: WHO.

3. World Health Organization (2013) Guideline: Calcium Supplementation in Pregnant Women. Geneva: WHO.

4. von Grebmer K, Saltzman A, Birol E et al. (2014) Global Hunger Index. Washington, DC: International Food Policy Research Institute.

5. Bloem MW, de Pee S, Hop le T et al. (2013) Key strategies to further reduce stunting in Southeast Asia: lessons from the ASEAN countries workshop. Food Nutr Bull 34, 2 Suppl., S8-S16.
6. National Institute of Statistics \& ICF Macro (2011) Cambodia Demographic and Health Survey. Calverton, MD: ICF Macro.

7. National Nutrition Program, Cambodia (2009) National Nutrition Training Curriculum - Minimum Package of Activities 10. Phnom Penh: Ministry of Health.

8. Ministry of Health, Cambodia (2011) National Policy and Guidelines for Micronutrient Supplementation to Prevent and Control Deficiencies in Cambodia. Phnom Penh: Ministry of Health.

9. Anon. (2011) Scaling Up Nutrition: A Framework for Action. http://scalingupnutrition.org/wp-content/uploads/2013/05/ SUN_Framework.pdf (accessed April 2015).

10. World Food Programme, Cambodia (2013) Maternal and Child Health and Nutrition Guidelines. Phnom Penh: WFP.

11. US Agency for International Development (2014) Corn Soy Blend/Plus Commodity Fact Sheet. http://www.usaid.gov/ what-we-do/agriculture-and-food-security/food-assistance/ resources/implementation-tools/corn-soy (accessed April 2015).

12. Nesbitt HJ (editor) (1997) Rice Production in Cambodia. Manila: International Rice Research Institute.

13. Ivankova NV, Creswell JW \& Stick SL (2006) Using mixedmethods sequential explanatory design: from theory to practice. Field Methods 18, 3-20.

14. Mack N, Woodsong C, Macqueen KM et al. (2005) Qualitative Research Methods: A Data Collector's Field Guide. Research Triangle Park, NC: Family Health International.

15. Kitzinger J (1995) Qualitative research: introducing focus groups. BMJ 311, 299-302.

16. Boeije H (2002) A purposeful approach to the constant comparative method in the analysis of qualitative interviews. Qual Quant 36, 391-409.

17. Young SL, Blanco I, Hernandez-Cordero S et al. (2010) Organoleptic properties, ease of use, and perceived health effects are determinants of acceptability of micronutrient supplements among poor Mexican women. J Nutr 140, 605-611.

18. World Bank (2015) Poverty Overview. http://www.worldbank. org/en/topic/poverty/overview (accessed April 2015).

19. de Pee S \& Bloem MW (2009) Current and potential role of specially formulated foods and food supplements for preventing malnutrition among 6- to 23-month-old children and for treating moderate malnutrition among 6- to 59-month-old children. Food Nutr Bull 30, 3 Suppl., S434-S463.

20. Pell C, Straus L, Andrew EV et al. (2011) Social and cultural factors affecting uptake of interventions for malaria in pregnancy in Africa: a systematic review of the qualitative research. PLoS One 6, e22452.

21. Huybregts LF, Roberfroid DA, Kolsteren PW et al. (2009) Dietary behaviour, food and nutrient intake of pregnant women in a rural community in Burkina Faso. Matern Child Nutr 5, 211-222.

22. Kumar R, Aslesh OP, Kumar A et al. (2013) Urban poor women do not increase their diet during pregnancy: a study from an urban resettlement colony in Delhi, India. Int J Med Public Health 3, 134-139.

23. de Sa J, Bouttasing N, Sampson L et al. (2013) Identifying priorities to improve maternal and child nutrition among the Khmu ethnic group, Laos: a formative study. Matern Child Nutr 9, 452-466.

24. Shannon K, Mahmud Z, Asfia A et al. (2008) The social and environmental factors underlying maternal malnutrition in rural Bangladesh: implications for reproductive health and nutrition programs. Health Care Women Int 29, 826-840.

25. Setyowati (2010) An ethnography study of nutritional conditions of pregnant women in Banten Indonesia. Makara Kesebatan 14, 5-10. 
26. Essén B, Johnsdotter S, Hovelius B et al. (2000) Qualitative study of pregnancy and childbirth experiences in Somalian women resident in Sweden. BJOG 107, 1507-1512.

27. Ali E, Zachariah R, Shams Z et al. (2015) Peanut-based ready-to-use therapeutic food: how acceptable and tolerated is it among malnourished pregnant and lactating women in Bangladesh? Matern Child Nutr 11, 1028-1035.

28. Rowe JP, Brodegard WC, Pike OA et al. (2008) Storage, preparation, and usage of fortified food aid among Guatemalan, Ugandan, and Malawian beneficiaries: a field study report. Food Nutr Bull 29, 213-220.

29. Adu-Afarwuah S, Lartey A, Zeilani M et al. (2011) Acceptability of lipid-based nutrient supplements (LNS) among
Ghanaian infants and pregnant or lactating women. Matern Child Nutr 7, 344-356.

30. Mridha MK, Chaparro CM, Matias SL et al. (2012) Acceptability of Lipid-Based Nutrient Supplements and Micronutrient Powders among Pregnant and Lactating Women and Infants and Young Children in Bangladesh and Their Perceptions about Malnutrition and Nutrient Supplements. Washington, DC: FHI 360/FANTA-2 Bridge.

31. Grimm P (2010) Social desirability bias. In Wiley International Encyclopedia of Marketing, vol. 2, p. 1 [J Sheth and NK Malhotra, editors-in-chief]. Hoboken, NJ: John Wiley \& Sons, Inc. 